\title{
Swim training and the genetic expression of adipokines in monosodium glutamate- treated obese rats
}

Paulo Vinicius Svidnicki', Nayara Carvalho Leite ${ }^{2}$, Marcelo Ricardo Vicari', Mara Cristina de Almeida', Roberto Ferreira Artoni' Giovani Marino Favero², Sabrina Grassiolli², Viviane Nogaroto'

1 Department of Structural, Molecular and Genetic Biology, Universidade Estadual de Ponta Grossa (UEPG) Ponta Grossa, PR, Brazil ${ }^{2}$ Department of General Biology, UEPG, Ponta Grossa, PR, Brazil

\author{
Correspondence to: \\ Viviane Nogaroto \\ Departamento de Biologia Estrutural \\ Molecular e Genética, Universidade \\ Estadual de Ponta Grossa \\ Av. General Carlos Cavalcanti, 4748 \\ 84030-900 - Ponta Grossa, PR, Brazil \\ vivianenogaroto@hotmail.com \\ Received on June/3/2014 \\ Accepted on Sept/14/2014
}

DOI: $10.1590 / 2359-3997000000039$

\begin{abstract}
Objective: The aim of this study was to evaluate the genetic expression of adipokines in the adipocytes of monosodium glutamate (MSG)-treated obese rats submitted to physical activity. Materials and methods: Obesity was induced by neonatal MSG administration. Exercised rats (MSG and control) were subjected to swim training for 30 min for 10 weeks, whereas their respective controls remained sedentary. Total RNA was obtained from sections of the mesenteric adipose tissue of the rats. mRNA levels of adiponectin (Adipoq), tumor necrosis factor alpha (Tnf), peroxisome proliferatoractivated receptor alpha (Ppara), and peroxisome proliferator-activated receptor gamma (Pparg) adipokines were quantified by quantitative Real-Time Polymerase Chain Reaction (qRT-PCR). Results: In the exercise-trained control group, the expression of Adipoq increased compared to the sedentary control, which was not observed in the MSG-obese rats. Increased levels of Tnf in MSG-obese rats were not reversed by the swim training. The expression of Ppara was higher in sedentary MSGobese rats compared to the sedentary control. Swimming increased this adipokine expression in the exercise-trained control rats compared to the sedentary ones. mRNA levels of Pparg were higher in the sedentary MSG-rats compared to the sedentary control; however, the exercise did not influenced its expression in the groups analyzed. Conclusions: In conclusion, regular physical activity was not capable to correct the expression of proinflammatory adipokines in MSG-obese rat adipocytes. Arch Endocrinol Metab. 2015;59(3):210-4
\end{abstract}

Keywords

Adipoq; Ppara; Pparg; quantitative RT-PCR; Tnf

\section{INTRODUCTION}

$\mathrm{N}$ eonatal treatment with monosodium glutamate (MSG) causes lesions in the arcuate nucleus, deregulating an important area involved in the body weight regulation and glycemic control $(1,2)$. Rats treated with MSG in the first days after birth develop obesity in adult life and present insulin resistance, glucose intolerance, dyslipidemia and cardiovascular dysfunction, which are typical symptoms of metabolic syndrome carriers (3). Similar to obese subjects, MSGobese rats present low-grade chronic inflammation, in particular on adipocyte tissue. Studies demonstrated that adipocyte-derived adipokine profile of MSG-obese rats is altered, thereby contributing to insulin resistance in this obesity model (4).

Regular physical activity is an important tool in the obesity control and associated comorbidities, impro- ving inflammatory condition typical of an obese individual. There are no studies with this animal model (MSG-obese rats) reporting the adipokines expression in mesenteric fat and physical activity. Thus, the aim of this study was to analyze the genetic expression profile of some adipokines of the mesenteric adipose tissue of MSG-treated obese rats subjected to swimming.

\section{MATERIALS AND METHODS}

Experiments were approved by the Ethical Committee for Animal Experiments of Universidade Estadual de Ponta Grossa (protocol number 02860). The obese group received subcutaneous injections of MSG ( $4 \mathrm{mg} / \mathrm{g}$ of body weight) during the first 5 days of life, whereas the control group received equimolar saline, a protocol adapted by (5-7). At the age of 21 days, ani- 
mals were weaned and divided in four groups $(\mathrm{N}=8$ rats per group): sedentary controls (CON-SED), exercisetrained controls (CON-EXE), sedentary MSG-treated animals (MSG-SED), and exercise-trained MSG-treated animals (MSG-EXE). All of the exercise-trained animals were subjected to regular swimming 3 times per week and $30 \mathrm{~min}$ per day for 10 weeks, according to (5). Each rat had a load weight equivalent to $5 \%$ of body mass attached to the base of tail to ensure that animals were in constant swimming activity. Araujo and cols. (8) adapted the lactate minimum test to swimming in rats. Blood lactate concentrations of $5.5 \mathrm{mM}$ are achieved at loads equivalent to $5 \%$ body weight, so this swimming program can be considered moderate exercise (8). The CON-SED and MSG-SED groups remained sedentary.

At the age of 90 days, the naso-anal length (NAL) and body weight were measured to assess the obesity degree in the animals using the Lee index $\left[{ }^{3} \sqrt{ }\right.$ (body weight $(\mathrm{g}) /$ naso-anal length $(\mathrm{cm})]$, which is an indicator of obesity used for rodents (9). Additionally, visceral fat (mesenteric) deposits were removed, washed and weighed. Data are reported considering body weight ( $\mathrm{g} / 100 \mathrm{~g}$ of body weight). All values are reported as means \pm standard error $($ SEM). The Student T-test was used with significance of $\mathrm{P}<0.05$.

For the analysis of genetic expression by qRT-PCR, sections of mesenteric fat were used for total RNA extraction and, subsequently cDNA synthesis. The amplified adipokines were: Adipoq, Tnf, Ppara and Pparg. The adipokines primer sequences were synthetized according to (4). The $18 \mathrm{~S}$ rDNA was used as an internal control. Relative change in genetic expression was presented as $2^{-\Delta \Delta C t}(10)$.

\section{RESULTS}

According to table 1 , body weight and NAL were 27.7 and $13.4 \%$ lower in the MSG-SED group, respectively, compared to the CON-SED group $(\mathrm{P}<0.05)$. As for the Lee index, the value was $4.7 \%$ higher in the MSG-
SED animals when compared to the CON-SED ones $(\mathrm{P}<0.05)$. MSG-SED rats presented a significantly increase of $109.9 \%$ of mesenteric fat in comparison with CON-SED rats. Swimming had no effect in rat NAL. However, there was a reduction of approximately 8.7 and $6.7 \%$ of body weight and 18.5 and $20.6 \%$ of mesenteric fat accumulation in CON-EXE and MSG-EXE, respectively, when compared to their respective sedentary groups $(\mathrm{P}<0.05)$. Physical activity significantly reduced the Lee index in $4.2 \%$ only in animals treated with MSG compared to the MSG-SED.

There was no statistical difference in the expression of Adipoq between MSG-SED and CON-SED groups. The expression level of adiponectin was approximately 6-fold higher in CON-EXE rats compared to the CON-SED rats $(\mathrm{P}<0.05)$ (Figure $1 \mathrm{~A})$. Tnf expression significantly increased approximately 4 times in the MSG-SED group in comparison with the CON-SED group. Nevertheless, in both groups, exercise did not alter the expression of $T n f$ in the mesenteric adipose tissue (Figure 1B). The expression levels of Ppara were on average 3.7 times higher in the MSG-SED group in comparison with the CON-SED group $(\mathrm{P}<0.05)$. The practice of physical activity resulted in a 4 .1-fold increase in the expression of Ppara in CON-EXE rats when compared to the CON-SED rats $(\mathrm{P}<0.05)$ (Figure IC). The levels of Pparg mRNA were around 4.5 times higher in the MSG-SED group, compared to the CON-SED $(\mathrm{P}<0.05)$. However, exercise did not influenced the expression of Pparg in the groups assessed (Figure 1D).

\section{DISCUSSION}

Rodents treated with MSG develop several obesity characteristics (1-3), probably caused by the reduction in the energy metabolism (11) and a dysfunction in the autonomic nervous system, which is characterized by a high parasympathetic and a low sympathetic activities, as consequences of hypothalamic lesion caused by MSG (12).

Table 1. Biometric parameters in MSG-obese and control rats submitted at swimming training

\begin{tabular}{|c|c|c|c|c|}
\hline Parameters & CON-SED & CON-EXE & MSG-SED & MSG-EXE \\
\hline Body weight (g) & $359.5 \pm 3.79^{\mathrm{c}, \mathrm{d}}$ & $328.4 \pm 7.7^{d}$ & $260.0 \pm 9.8^{\mathrm{a}}$ & $242.6 \pm 11.3^{a, b}$ \\
\hline NAL (cm) & $23.1 \pm 0.1^{c, d}$ & $22.9 \pm 0.3^{\mathrm{c}, \mathrm{d}}$ & $20.0 \pm 0.5^{a, b}$ & $20.4 \pm 0.5^{\mathrm{a}, \mathrm{b}}$ \\
\hline Lee index & $0.298 \pm 0.002^{c}$ & $0.283 \pm 0.013^{\mathrm{c}, \mathrm{d}}$ & $0.312 \pm 0.003^{a, b, d}$ & $0.299 \pm 0.005^{b, c}$ \\
\hline Mesenteric fat $(\mathrm{g} / 100 \mathrm{~g})$ & $0.81 \pm 0.05^{c, d}$ & $0.66 \pm 0.03^{c, d}$ & $1.70 \pm 0.12^{a, b, d}$ & $1.35 \pm 0.10^{a, b, c}$ \\
\hline
\end{tabular}

Data are presented as mean \pm SEM obtained from 8 rats by group. Letters above of numbers represent statistical difference $(P<0.05)$ in Student T-test. ${ }^{a}$ CON-SED; ${ }^{b}$ CON-EXE; ${ }^{c}$ MSG-SED; ${ }^{\circ}$ MSG-EXE. 

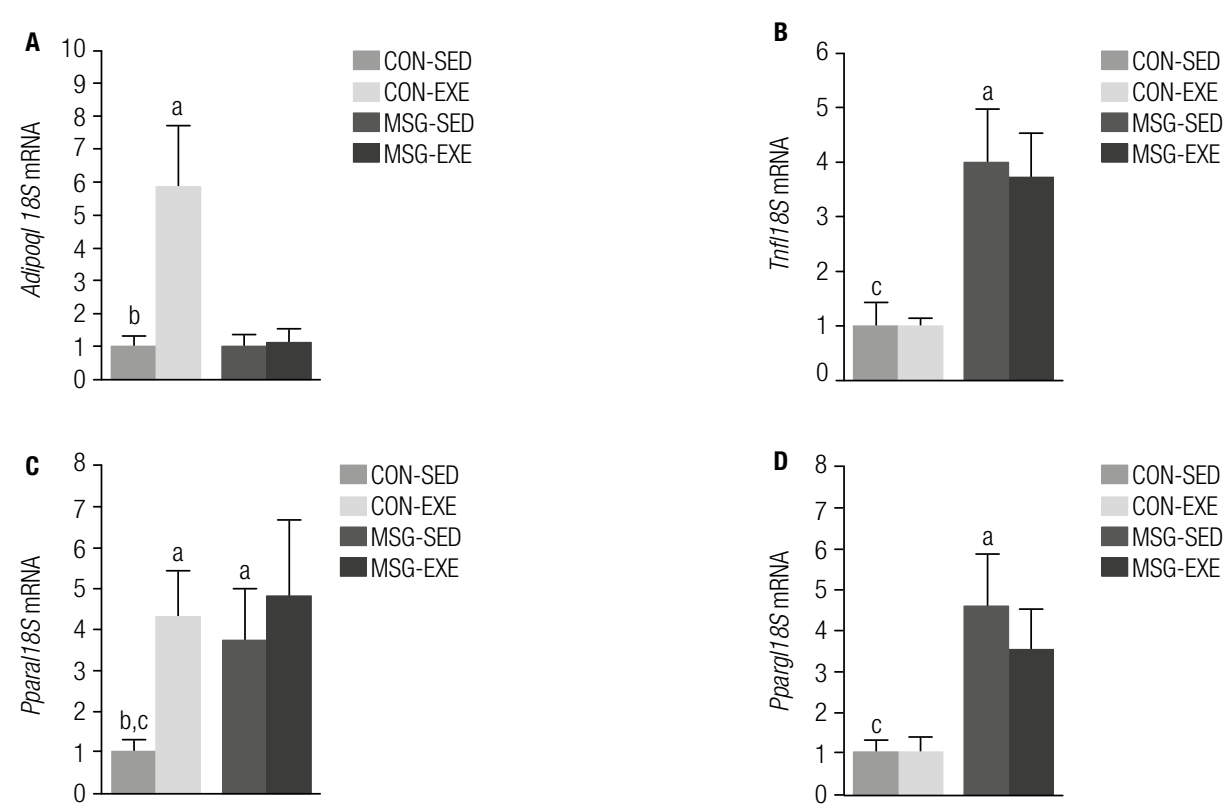

Figure 1. mRNA expression levels of the mesenteric fat tissue samples of MSG-obese and control rats submitted at swimming training ( $\mathrm{N}=8$ rats per group). (A) Adipoq, (B) Tnf, (C) Ppara and, (D) Pparg. Letters above the bars represent statistical difference in Student T-test (P<0.05). ${ }^{a}$ CON-SED; ${ }^{\mathrm{b}} \mathrm{CON}-\mathrm{EXE} ;{ }^{\mathrm{c}} \mathrm{MSG}-\mathrm{SED} ;{ }^{\mathrm{d}} \mathrm{MSG}-\mathrm{EXE}$.

The increase in the Lee index associated with a major accumulation of mesenteric fat in MSG-treated obese rats confirmed the efficacy of MSG neonatal administration in obesity induction in the animals of our study. However, MSG-obese rats presented reduction in body weight and in NAL due to the delay in bone and muscle development, which is a consequence of a reduction in the growth hormone $(\mathrm{GH})$ levels resulting from the arcuate nucleus lesion (13).

Corroborating with previous reports $(5,6)$, our study showed that swimming $30 \mathrm{~min} / 3$ times per week with a load weight equivalent to $5 \%$ of body mass was effective in promoting reduction in mesenteric fat deposits in MSG-treated animals, without causing collateral effects. Regular swimming corrects hyperinsulinemia and resistance to insulin and favors lipolysis triggered by the activation of the sympathetic nervous system (5). Similarly, Shima and cols. (14) using run in rats showed that frequency of 2 to 3 times/week is sufficient to prevent development of type 2 diabetes in OLETF rats (spontaneous model of type 2 diabetes). The training protocol used in this study began immediately after weaning, when rodents may be treated in terms of development like children. Atlantis and cols. (15) revised the beneficial effects of body fat reduction in obesity/overweight children submitted to exercise and showed that good results in training can be ob- tained with exercises in lower intensity and frequency, compared with those recommended in the literature.

Regular physical activities, even if there is no loss of weight, are related to a substantially reduction of total and visceral fat, besides leading to a significantly improvement in obesity $(16,17)$. MSG-SED rats have hypertrophic adipocytes (5), which presented an altered inflammatory profile directly related to insulin resistance. Carvalho Leite and cols. (5) demonstrated that swim training program was effective in attenuating morphological alterations in the adipose tissue and pancreatic islets in MSG-treated obese rats. Histological analyzes in adipose tissue deposits, demonstrated that physical training reduced the adipocyte diameter and increased the number of adipocytes in MSG-EXE and CON-EXE groups, compared with their sedentary counterparts (5).

It is known that the expression of adiponectin mRNA is inversely related to obesity, type 2 diabetes mellitus (T2DM) and cardiovascular diseases (18). Our study showed that there was no difference in adiponectin expression between MSG-obese rats and control animals. Other studies have already shown that MSG obesity model did not reflect hyperadiponectinemia or adiponectin resistance, a phenomenon observed in other obesity models $(4,19)$. Our research showed that swimming stimulated the genetic expression of adiponectin in adipocytes of CON-EXE rats. High plasma 
levels of adiponectin were also found in humans subjected to aerobic exercises (20). On the other hand, exercised MSG-obese rats did not present alteration in adiponectin expression, which may reinforce that adiponectin levels in the MSG model is normal.

MSG rats presented a higher expression of Tnf, when compared to control animals, corroborating with Roman-Ramos and cols. (4). Indeed, expression levels of Tnf increased in obesity, T2DM and cardiovascular disease conditions (18). TNF promotes reduction in adiponectin expression and secretion (18), contributing to metabolic syndrome development. Together, these data indicate that high levels of Tnf expression found in adipocytes of MSG-obese rats are probably due to chronic inflammation of the adipose tissue with macrophage infiltration (21). Probably this adipokine contributes to insulin resistance, which is typical for this model. According to our data, exercise does not correct the inflammatory profile in this obesity model.

Corroborating Roman-Ramos and cols. (4), Ppara and Pparg had their mRNA levels increased in the MSG-treated animals, which may indicate an inflammatory condition due to obesity. Pparg is mainly expressed in the adipose tissue and its activation in MSG-treated rat adipose tissue may lead to the increase of adipocyte size, probably causing hypertrophy (5). Roman-Ramos and cols. (4) suggested that the activation of PPARs is responsible for the deregulated inflammatory profile of MSG animals; however, some authors reported that Ppara and Pparg activation may improve insulin resistance induced by obesity (22). Our results indicate that Pparg may participate directly in the increase of the visceral fat content and adipocyte size that occurs in MSG-obese animals (5). On the other hand, exercise was not able to correct its levels in obese animals. Probably, Pparg function is not only metabolic, but it may also be involved in the inflammatory control, participating in the proinflammatory cytokine pathway and in the modulation of acute phase of the inflammatory response by independent mechanisms of lipoprotein changes (23).

Genes regulated by Ppara participate in the key-protein regulation involved in lipid metabolism, fatty acid oxidation, hemostasis and inflammation (24). High levels of Ppara mRNA may be related to the increase of body weight in MSG-treated animals. Physical activity did not have influence over this gene expression in these animals. The higher expression of Ppara mRNA in CON-EXE animals can be explained by a high mi- tochondrial activity and peroxisomal beta-oxidation. PPARA is capable of upregulating the expression of genes to enzymes such as carnitine-palmitoyltransferase-I (CPT-I), which transports fatty acids into the mitochondria for beta-oxidation. Expression of PPARA also increases expression of acetyil-CoA synthase, a mitochondrial enzyme necessary for beta-oxidation (25). However, more studies are needed to determine which exactly the role of Ppara is and its relation to obesity.

Regular physical activity was not capable to correct proinflammatory adipokine expression in adipocytes of MSG-treated obese rats. Other factors or adipokines cannot be discarded. More studies are necessary to determine which adipokines have altered expression by physical activity and the resulting effect in energy homeostasis. Furthermore, studies to analyze the resulting proteins of the analyzed genes are necessary, although cytokines may have autocrine and paracrine actions, which may affect the metabolism of adipose tissue without necessarily affecting plasma levels.

Acknowledgments: this study was financed by Coordenação de Aperfeiçoamento de Pessoal de Nível Superior (Capes), Fundação Araucária de Apoio ao Desenvolvimento Científico e Tecnológico do Estado do Paraná (Fundação Araucária), Conselho Nacional de Desenvolvimento Científico e Tecnológico (CNPq) and Secretaria de Estado da Ciência, Tecnologia e Ensino Superior/ Unidade Gestora do Fundo do Paraná (SETI/UGF).

Disclosure: no potential conflict of interest relevant to this article was reported.

\section{REFERENCES}

1. Burde RM, Schanker B, Kayes J. Acute effect of oral and subcutaneous administration of monosodium glutamate on the arcuate nucleus of the hypothalamus in mice and rats. Nature. 1971;233(5314):58-60.

2. Schwartz MW, Porte D Jr. Diabetes, obesity, and the brain. Science. 2005;307(5708):375-9.

3. Elfers C, Ralston M, Roth CL. Studies of different female rat models of hypothalamic obesity. J Pediatr Endocrinol Metab. 2011;24(3-4):131-7.

4. Roman-Ramos R, Almanza-Perez JC, Garcia-Macedo R, BlancasFlores G, Fortis-Barrera A, Jasso El, et al. Monosodium glutamate neonatal intoxication associated with obesity in adult stage is characterized by chronic inflammation and increased mRNA expression of peroxisome proliferator-activated receptors in mice. Basic Clin PharmacolToxicol. 2011;108(6):406-13.

5. Carvalho Leite N, Ferreira TR, Rickli S, Borck PC, Mathias PC, Emilio HRO, et al. Glycolytic and mitochondrial metabolism in pancreatic islets from MSG-treated obese rats subjected to swimming training. Cell Physiol Biochem. 2013;31(2-3):242-56.

6. Svidnicki PV, de Carvalho Leite N, Venturelli AC, Camargo RL, Vicari MR, de Almeida MC, et al. Swim training restores glucagon-like peptide-1 insulinotropic action in pancreatic islets from monosodium glutamate-obese rats. Acta Physiol (Oxf). 2013;209(1):34-44. 
7. Grassiolli S, Gravena C, de Freitas Mathias PC. Muscarinic M2 receptor is active on pancreatic islets from hypothalamic obese rat. Eur J Pharmacol. 2007;556(1-3):223-8.

8. de Araujo GG, Papoti M, Manchado Fde B, de Mello MA, Gobatto CA. Protocols for hyperlactatemia induction in the lactate minimum test adapted to swimming rats. Comp Biochem Physiol A Mol Integr Physiol. 2007;148(4):888-92.

9. Bernardis LL, Patterson BD. Correlation between "Lee index" and carcass fat content in weanling and adult female rats with hypothalamic lesions. J Endocrinol. 1968;40(4):527-8.

10. Livak KJ, Schmittgen TD. Analysis of relative gene expression data using real-time quantitative PCR and the 2(-Delta Delta C(T) Method. Methods. 2001;25(4):402-8.

11. Tsukahara F, Uchida Y, Ohba K, Ogawa A, Yoshioka T, Muraki T. The effect of acute cold exposure and norepinephrine on uncoupling protein gene expression in brown adipose tissue of monosodium glutamate-obese mice. Jpn J Pharmacol. 1998;77(3):247-9.

12. Balbo SL, Mathias PC, Bonfleur ML, Alves HF, Siroti FJ, Monteiro OG, et al. Vagotomy reduces obesity in MSG-treated rats. Res Commum Mol Pathol Pharmacol. 2000;108(5-6):291-6.

13. Tamura H, Kamegai J, Shimizu T, Ishii S, Sugihara H, Oikawa S. Ghrelin stimulates $\mathrm{GH}$ but not food intake in arcuate nucleus ablated rats. Endocrinol. 2002;143(9):3268-75.

14. Shima K, Shi K, Mizuno A, Sano T, Ishida K, Yoshimoto S. Effects of difference in amount of exercise training on prevention of diabetes-mellitus in the Otsuka-Long-Evans-Tokushima fatty rats, a model of spontaneous non-insulin-dependent diabetes mellitus. Diabetes Res Clin Pract. 1994;23(3):147-54.

15. Atlantis $E$, Barnes EH, Singh MA. Efficacy of exercise for treating overweight in children and adolescents: a systematic review. Int J Obes (Lond). 2006;30(7):1027-40.
16. Lee S, Kuk JL, Davidson LE, Hudson R, Kilpatrick K, Graham TE, et al. Exercise without weight loss is an effective strategy for obesity reduction in obese individuals with and without type 2 diabetes. J Appl Physiol (1985). 2005;99(3):1220-5.

17. Linden MA, Pincu Y, Martin SA, Woods JA, Baynard T. Moderate exercise training provides modest protection against adipose tissue inflammatory gene expression in response to high-fat feeding. Physiol Rep. 2014;2(7):pii: e12071.

18. Hutley L, Prins JB. Fat as an endocrine organ: relationship to the metabolic syndrome. Am J Med Sci. 2005;330(6):280-9.

19. Segersvärd R, Tsai JA, Herrington MK, Wang F. Obesity alters cytokine gene expression and promotes liver injury in rats with acute pancreatitis. Obesity (Silver Spring). 2008;16(1):23-8.

20. Kriketos AD, Gan SK, Poynten AM, Furler SM, Chisholm DJ, Campbell LV. Exercise increases adiponetin levels and insulin sensitivity in humans. Diabetes Care. 2004;27(2):629-30.

21. Weisberg SP, McCann D, Desai M, Rosenbaum M, Leibel RL, Ferrante AW Jr. Obesity is associated with macrophage accumulation in adipose tissue. J Clin Invest. 2003;112(12):1796-808.

22. Tavares $\mathrm{V}$, Hirata $\mathrm{MH}$, Hirata RD. [Peroxisome proliferator-activated receptor gamma (PPARgamma): molecular study in glucose homeostasis, lipid metabolism and therapeutic approach]. Arq Bras Endocrinol Metab. 2007;51(4):526-33.

23. Semple RK, Chatterjee VK, O'Rahilly S. PPAR gama and human metabolic disease. J Clin Invest. 2006;116(3):581-9.

24. Torra IP, Chinetti G, Duval C, Fruchart JC, Staels B. Peroxisome proliferator-activated receptors: from transcriptional control to clinical practice. Curr Opin Lipidol. 2001;12(3):245-54.

25. Mascaró C, Acosta E, Ortiz JA, Marrero PF, Hegardt FG, Haro D. Control of human muscle-type carnitine palmitoyltransferase I gene transcription by peroxisome proliferator-activated receptor. J Biol Chem. 1998;273(15):8560-3. 